HELMINTHOLOGIA, 52, 3: 205 - 210, 2015

\title{
Serological survey for canine angiostrongylosis in Slovakia
}

\author{
M. MITERPÁKOVÁ ${ }^{*}$, M. SCHNYDER ${ }^{2}$, R. SCHAPER ${ }^{3}, Z$. HURNÍKOVÁ 1,4, V. ČABANOVÁ 1
}

1'nstitute of Parasitology, Slovak Academy of Sciences, Hlinkova 3, 04001 Košice, Slovakia, ${ }^{*} E-m a i l:$ miterpak@saske.sk; ${ }^{2}$ Institute of Parasitology, University of Zurich, Winterthurerstr. 266a, CH-8057 Zurich, Switzerland; ${ }^{3}$ Bayer Animal Health GmbH, Monheim, 6210, 51368 Leverkusen, Germany; ${ }^{4}$ University of Veterinary Medicine and Pharmacy, Komenského 73, 04001 Košice, Slovakia

\section{Article info}

Received February 5, 2015 Accepted March 17, 2015

\begin{abstract}
Summary
In recent years Angiostrongylus vasorum has become another important heart parasite of dogs besides Dirofilaria immitis, with intense spread into new areas of Europe. The first two cases of canine angiostrongylosis in Slovakia were observed in 2013, demonstrating that this life-threatening parasitic disease of dogs has expanded into this territory too. One year after the first $A$. vasorum findings, a serological survey was conducted to assess the current distribution of this parasitic infection in dogs from Slovakia.

Serum samples from 225 dogs were collected from 29 veterinary practices situated in 22 districts of Slovakia and tested by ELISA for the presence of circulating $A$. vasorum antigens and additionally for the detection of specific antibodies against $A$. vasorum.

Fourteen samples (6.22\%) were seropositive in at least one ELISA. Of these, 7 dogs $(3.11 \%)$ were only antibody-positive and 4 dogs $(1.78 \%)$ were positive only for circulating $A$. vasorum antigen. Three animals out of 225 examined (1.33 \%) were positive in both ELISAs.

Keywords: Angiostrongylus vasorum; dogs; ELISA; antigen and antibody detection; seroprevalence; Slovakia
\end{abstract}

\section{Introduction}

Canine angiostrongylosis, caused by the metastrongylid nematode Angiostrongylus vasorum, was established in dogs for a long time in endemic areas of France, Denmark, southern England, Wales and Ireland. In more recent years its spread into new areas of Europe has been demonstrated. The first two autochthonous cases of canine angiostrongylosis in Slovakia were observed in 2013 (Hurníková et al., 2013; Miterpáková et al., 2014), showing that this life-threatening parasitic disease of dogs has expanded into this territory too.

Given that the parasite has also been diagnosed in dogs in the neighbouring countries Hungary (e.g. Majoros et al., 2010; Tolnai et al., 2015) and the Czech Republic (Hajnalová et al., 2014), and also during a serological survey in Poland (Schnyder et al., 2013b), a serological survey was performed to assess the current distribution of this parasitic infection in dogs from Slovakia.

\section{Materials and Methods}

Serum samples from 225 dogs were collected from 29 veterinary practices situated in 22 districts of Slovakia. The veterinarians were asked to choose dogs randomly, and individual data related to age, breed, sex, locality of residence, living habitat and health status were collected in a questionnaire. All samples were delivered to the Institute of Parasitology (Slovak Academy of Sciences, Košice, Slovakia).

Serum samples were tested by ELISA for the presence of circulating $A$. vasorum antigens using mono-and polyclonal antibodies as previously described, with a sensitivity of $95.7 \%$ and a specificity of $98.8 \%$ (Schnyder et al., 2011), and additionally for the detection of specific antibodies against $A$. vasorum in a sandwich-ELISA using $A$. vasorum adult somatic antigen purified by monoclonal antibodies (mAb Av 5/5), with a sensitivity of $81.0 \%$ and a specificity of $98.8 \%$ (Schucan et al., 2012), at the Institute of Parasitology, 
Table 1. Dog sera from Slovakia $(n=225)$ tested for the presence of circulating Angiostrongylus vasorum antigen and specific antibodies against Angiostrongylus vasorum

\begin{tabular}{lcc}
\hline \multicolumn{1}{c}{$\mathrm{n}=\mathbf{2 2 5}$} & Number positive & $\%$ (95\% confidence interval) \\
\hline Total seropositive samples & 14 & $6.22( \pm 3.16)$ \\
Seropositive for antibodies and antigen & 3 & $1.33( \pm 1.5)$ \\
Seropositive for antibody & 10 & $4.44( \pm 2.69)$ \\
Seropositive for antibodies only & 7 & $3.11( \pm 2.27)$ \\
Seropositive for antigen & 7 & $3.11( \pm 2.27)$ \\
Seropositive for antigen only & 4 & $1.78( \pm 1.73)$ \\
\hline
\end{tabular}

University of Zurich, Switzerland. All test runs included a background control, a conjugate control, three positive control sera from three experimentally infected dogs and two negative control sera from uninfected dogs.

The collected data were analyzed by a geographic information system (GIS) using the program RegioGraph 10 (GfK GeoMarketing, Bruchsal, Germany) to visualize the regional distribution of collected and analyzed serum samples and $A$. vasorum antigen- and/or antibody-positive samples. The locations of positive samples were displayed on maps with administrative and postcode boundaries based on the two-digit postcodes of Slovakia as points of reference.

The seroprevalence values were calculated with $95 \%$ confidence intervals $(\mathrm{Cl})$ using the STATISTICA 6 Base programme (StatSoft, Inc., 2001).

\section{Results}

The serological results of all tested samples are summarized in Table 1. Fourteen samples ( $6.22 \% ; \mathrm{Cl} \pm 3.16 \%$ ) were seropositive in at least one ELISA. Of these, 7 dogs (3.11\%) were only antibody-positive and 4 dogs $(1.78 \%)$ were positive only for circulating A. vasorum antigen. Three animals out of 225 examined $(1.33 \%)$ were positive in both ELISAs.

Seropositive dogs came from different regions of Slovakia, with the highest accumulation in the south-western part (Fig. 1). All three dogs positive for both circulating antigens and specific antibodies against $A$. vasorum lived in the Bratislava region - two in the city of Bratislava and one in the Austrian village of Deutsch Jahrndorf on the border between Hungary and Slovakia, roughly $20 \mathrm{~km}$ from Bratislava.

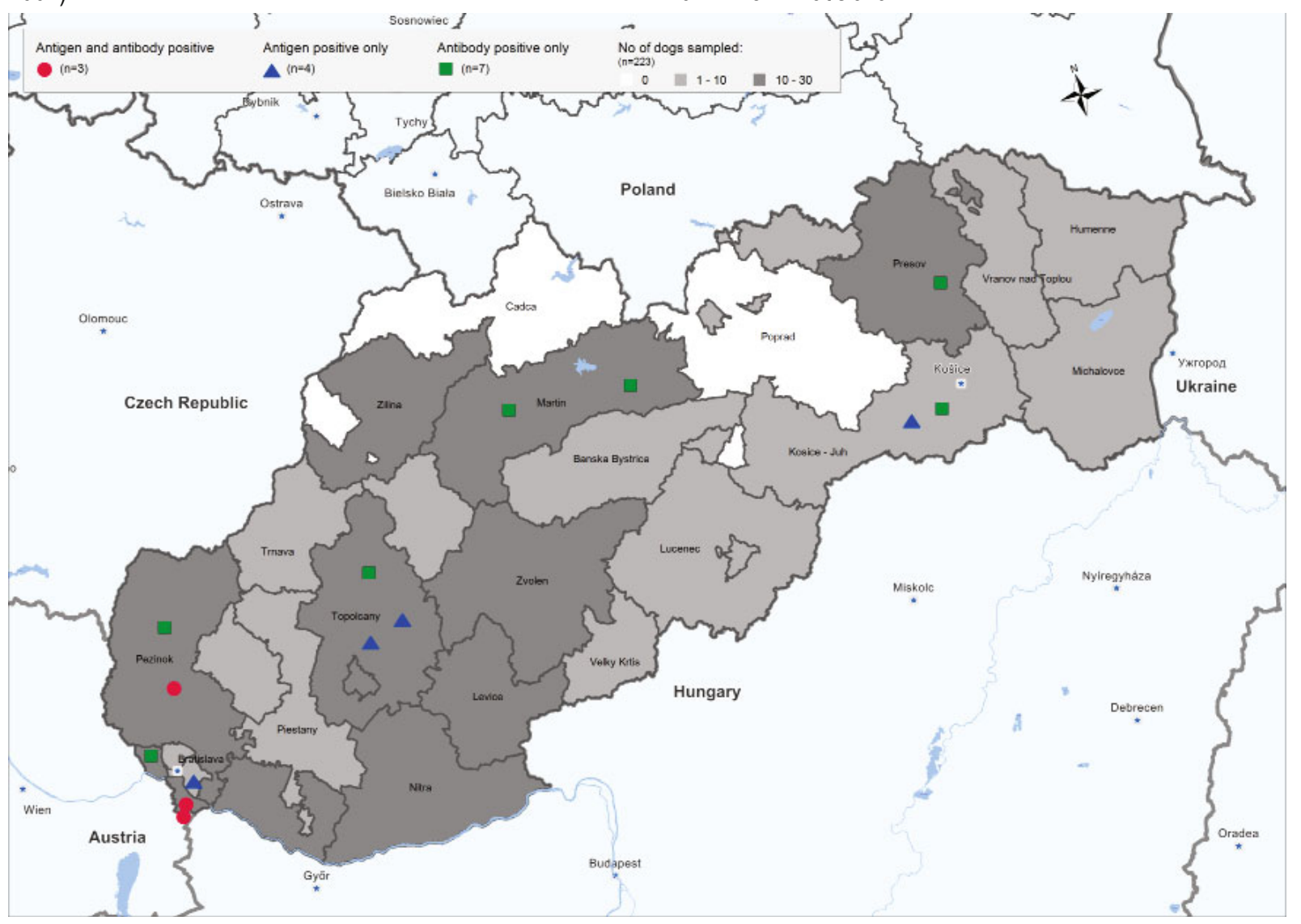

Figure 1. Distribution of dogs serologically positive for Angiostrongylus vasorum in Slovakia 


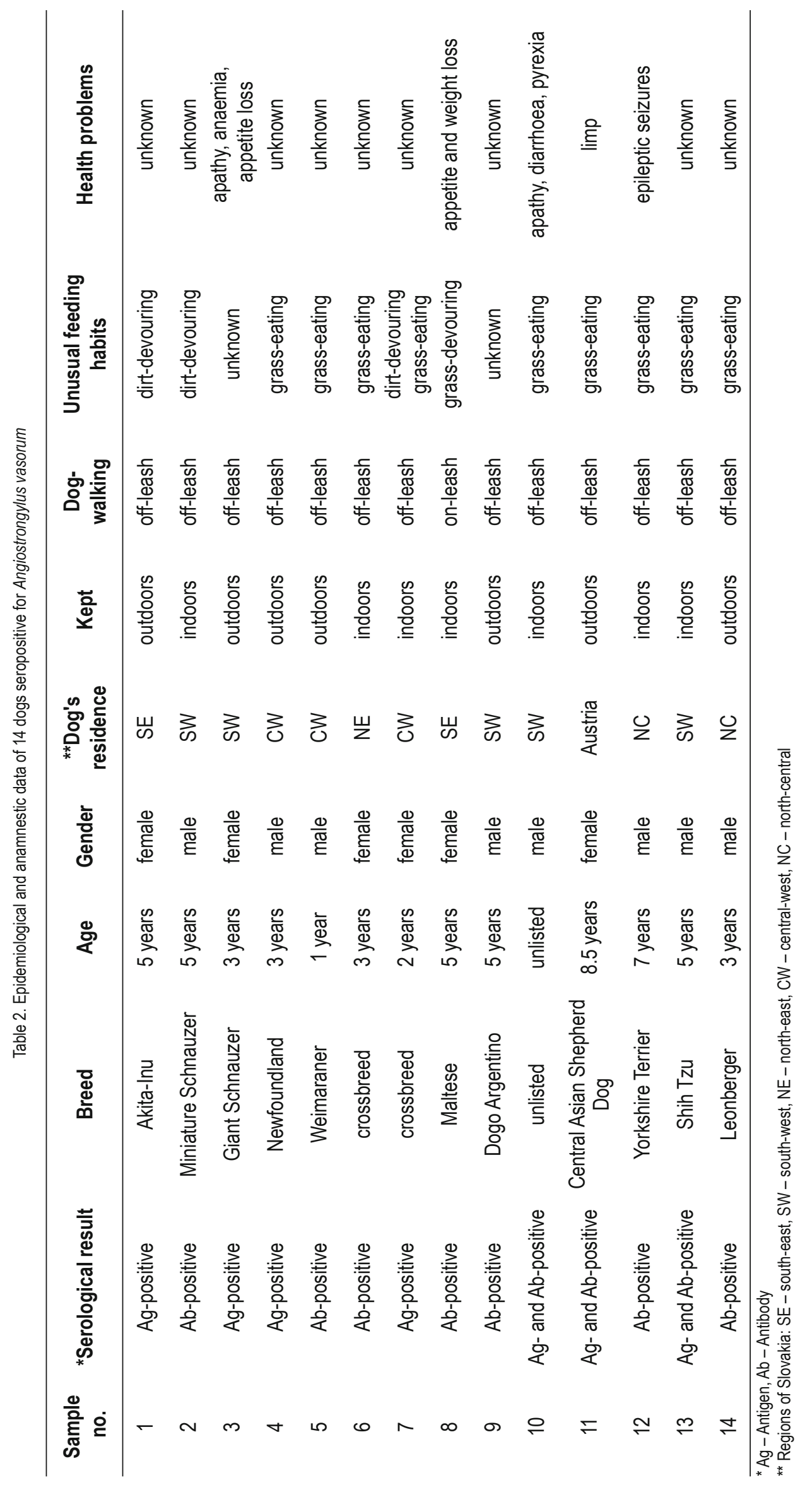


The analysis of epidemiological and anamnestic data contained in the questionnaires showed that the average age of seropositive dogs was 4.3 years with a range from 1 to 8.5 years. Among the positive dogs, large and small breeds were equally represented, and 8 individuals were males and 6 females. Seven of the dogs were kept outdoors and 7 indoors, and all owners but one reported that the dog moved outside mostly without restraint and leash. In ten of the seropositive dogs, grass feeding and also devouring other components in the environment, including waste and excreta, were frequently observed (Table 2).

From the medical point of view, 9 out of 14 seropositive dogs showed no clinical signs, but in three seropositive dogs signs potentially related to the $A$. vasorum infection were registered, including significant weight loss and lack of appetite, lethargy, epileptic seizures, anaemia, diarrhoea, and pyrexia.

\section{Discussion}

The serological survey on canine angiostrongylosis presented here was conducted one year after the first findings of Angiostrongylus vasorum in Slovakia confirmed by larval isolation with Baermann's method (Hurníková et al., 2013; Miterpáková et al., 2014). Our results indicated relatively high overall seropositivity $(6.22 \%)$, with $A$. vasorum circulating antigens and/or specific antibodies against the parasite being diagnosed in 14 of the 225 dogs examined. In total, $1.33 \%$ of the dogs $(3 / 255, \mathrm{Cl} \pm 1.5 \%)$ were positive in both ELISAs. By comparison, in a known endemic area such as southern England, only $0.97 \%$ of the dogs were seropositive for both ELISAs (Schnyder et al., 2013a). However, it needs to be emphasized that in that study more than 4,000 serum samples were tested. The results presented here are comparable with a recent serosurvey performed in Portugal, where the parasite was also discovered only recently: out of a total of 341 shelter dogs, $1.17 \%$ were seropositive for both ELISAs (Alho et al., 2014).

Although angiostrongylosis is considered to be an infection that more frequently affects dogs younger than one year (Chapman et al., 2004; Koch et al., 2005; Staebler et al. 2005; Barutzki and Schaper, 2009; Chapman et al., 2004; Staebler et al., 2005), our results confirmed that older individuals are also at risk. Interestingly, one single dog tested antibody-positive at the age of one year, while all others were older than 3 years and in particular the oldest dog, positive for the presence of both, antigens and antibodies, was 8.5 years old at the time of sampling (Table 2). Since $A$. vasorum infections may persist for a lifetime in untreated dogs (Guilhon and Cens, 1969) and occasionally remain unnoticed for a long time period (due to restricted or nonspecific clinical signs (Schnyder et al., 2010) or lack of disease awareness, especially in new endemic areas where $A$. vasorum has not previously been diagnosed), the time point of oral intake of the infectious stages in naturally infected dogs is unknown. Therefore, the time point at which the positive dogs in this study were infected is also unknown and may have occurred years before and/or on multiple different occasions.

In epidemiological terms, the relevant information is that all dogs but one were moving outdoors freely without leashes, and except for two, all owners of seropositive dogs reported that their dog of- ten ate grass or other environmental components. Given that the parasite's life cycle is indirect via snails (and possibly their secreta) (Ferdushy and Hasan, 2010) and perhaps paratenic hosts (Bolt et al., 1993; Mozzer and Lima, 2015), these feeding habits should be considered to be a major risk factor.

Regarding breed predisposition for angiostrongylosis, results from previous studies are inconclusive. Some authors did not report any contingency (Koch and Willesen, 2009; Staebler et al., 2005), while others suggest that the risk may be higher in purebred dogs or in the hunting dog breeds (Chapman et al., 2004; Conboy, 2004). Among the seropositive dogs detected within our study, 7 belonged to large breeds, 3 were small breeds and 3 individuals were of unascertained identity, confirming the trend of no breed predisposition.

From a clinical point of view it can be very difficult to identify clinical signs unambiguously related to $A$. vasorum infection, since the clinical picture of this disease is very diverse and can evoke a variety of other cardiopulmonary, neurological or haematological disorders or further nonspecific signs (Koch and Willesen, 2009; Capogna et al., 2012). In fact, the owners of three serologically positive dogs reported signs such as apathy, anaemia, and marked loss of weight and appetite, which are nonspecific but quite common in dogs infected with $A$. vasorum (Koch and Willesen, 2009).

The above-mentioned broad spectrum of clinical signs and a possible, at least initially, asymptomatic course of the disease may be reasons why veterinary practitioners may overlook the infection in patients, especially in areas where it has not previously occurred. Since standard microscopic methods for the isolation and identification of first larval stages of lungworms are actually rarely implemented in veterinary practices, a diagnosis may be missed, and more specific methods such as the Baermann funnel method need to be applied. Alternatively, a new commercially available antigen blood test (AngioDetect ${ }^{\circledR}$, Idexx Laboratories, USA) may be used, with which results can be obtained within 15 minutes. This test can detect an ongoing infection starting from 9 weeks after infection with a very high specificity of $100 \%(\mathrm{Cl}$ : 97.6 - $100 \%)$ (Schnyder et al., 2014), whereas the ELISAs employed in this study detect circulating antigens around 5 weeks post infection (Schnyder et al., 2011) and specific antibodies starting from 3 weeks post infection (Schucan et al., 2012), both also with very high specificity $(98.8 \%)$. In all three tests, including the two ELISAs used in this study, cross-reactivity against several helminthes, and in particular against the widespread lung worm Crenosoma vulpis and the less common heart worm Dirofilaria immitis was evaluated, delivering these high specificities. On the other hand, specific antibodies against $A$. vasorum and circulating antigens can persist for more than 9 and 4 weeks, respectively, after treatment (Schnyder et al., 2011; Schucan et al., 2012). Therefore, while the antigen-based rapid assay has a primary application in the diagnosis of dogs clinically suspect for canine angiostrongylosis, ELISA-based techniques are important tools for mass screening and monitoring of infections in non-endemic or newly infested regions. In particular in areas of low prevalence, the combination of both ELISAs for the detection of $A$. vasorum antigen and specific antibodies is of great value in terms of positive predictive value (Schnyder et al., 2013a). A number of factors influence the distribution of $A$. vasorum (Mor- 
gan et al., 2009). The parasite does not necessarily occur in large endemic areas, like Dirofilaria spp., but its presence is incoherent with hyperendemic foci surrounded by areas of low prevalence or localities with sporadic cases (Barutzki and Schaper, 2009; Guardone et al., 2013; Schnyder et al., 2013b). However, it is clear from recent studies that the geographic range of $A$. vasorum in Europe is changing and that the parasite has been identified in new areas distant from the original endemic foci in recent years. One of the new regions in which the occurrence of canine angiostrongylosis can be expected in the very near future is Central and Eastern Europe. In addition to Slovakia, serological screening was carried out in Poland in 3,345 dogs, revealing $1.79 \%$ of them to be antibody-positive and $0.51 \%$ concurrently antigen- and antibody-positive (Schnyder et al., 2013b). In Poland, which neighbours Slovakia to the north, $A$. vasorum larvae were also confirmed in wolves using Baermann's method (Szczęsna et al., 2007), and adult parasites were detected in the pericardium and pulmonary arteries of four foxes (Demiaszkiewicz et al., 2014). In Hungary, which borders Slovakia to the south, the presence of $A$. vasorum was confirmed in a dog and in the intermediate host Arion lusitanicus (Portuguese slug) in 2010 (Majoros et al., 2010) and also in wild carnivores such as foxes (Sreter et al., 2003) and golden jackals (Takacs et al., 2014) and is meanwhile broadly spread, also in the neighboring areas (Tolnai et al., 2015). In the Czech Republic, to the north-west of Slovakia, the first case of canine angiostrongylosis was reported in 2014 (Hajnalová et al., 2014). In Austria only imported cases of canine angiostrongylosis have been reported (Maier et al., 2010) up to now, but one of the seropositive dogs found during our survey came from the Lower Austrian village of Deutsch Jahrndorf on the Austria-Slovakia-Hungarian border. To the best of our knowledge, no data about the occurrence of $A$. vasorum in Ukraine (bordering eastern Slovakia) are available. Most recently, an autochthonous case of canine angiostrongylosis was diagnosed is Serbia (Simin et al., 2014) and it can be assumed that further cases will occur.

Further monitoring is required in order to evaluate the distribution of this parasitic disease in Slovakia. However, as our experience indicates, newly emerging diseases are associated with great difficulties such as determining the correct clinical diagnosis, the use of appropriate diagnostic tools and, correspondingly, the provision of adequate treatment by veterinary practitioners (Miterpáková et al., 2012). Efforts to increase awareness of this potentially fatal parasitic disease among animal owners and veterinary practitioners are therefore needed.

\section{References}

Alho, A.M., Schnyder, M., Meireles, J., Belo, S., Deplazes, P., MADEIRA DE CARVALho, L. (2014): Preliminary results on the seroprevalence of Angiostrongylus vasorum and co-infection with Dirofilaria immitis in shelter dogs from Portugal. Parasit. Vectors, 7: 026. DOI:10.1186/1756-3305-7-S1-O26

BARUTZKI, D., Schaper, R. (2009): Natural infections of Angiostrongylus vasorum and Crenosoma vulpis in dogs in Germany (2007 - 2009). Parasitol. Res., 105 Suppl 1: 39 - 48. DOI: 10.1007/ s00436-009-1494-x
Bolt, G., Monrad, J., Frandsen, F., Henriksen, P., Dietz, H.H. (1993): The common frog (Rana temporaria) as a potential paratenic and intermediate host for Angiostrongylus vasorum. Parasitol. Res., 79: $428-430$

Capogna, A., Sasanelli, M., Paolo, Lia, R.P., Spagnolo, P.P., Paradies, P. (2012): Further insights into the clinical aspects of Angiostrongylus vasorum natural infection in symptomatic and asymptomatic dogs. J. Vet. Med. Sci., 1:2. DOI: http://dx.doi. org/10.4172.2325-9590.1000106

Chapman, P.S., Boag, A.K., Guitian, J., Boswood, A. (2004): Angiostrongylus vasorum infection in 23 dogs $(1999-2002)$. J. Small Anim. Pract., 45: 435 - 440

Conboy, G. (2004): Natural infections of Crenosoma vulpis and Angiostrongylus vasorum in dogs in Atlantic Canada and their treatment with milbemycin oxime. Vet. Rec., 155: 16 - 18

Demiaszkiewicz, A.W., Pyziel, A.M., Kuligowska, I., Lachowicz, J. (2014): The first report of Angiostrongylus vasorum (Nematoda; Metastrongyloidea) in Poland in red foxes (Vulpes vulpes). Acta Parasitol., 59 (4): 758 - 762. DOI: 10.2478/s11686-014-0290-7

Ferdushy, T., Hasan, M.T. (2010): Survival of first stage larvae (L1) of Angiostrongylus vasorum under various conditions of temperature and humidity. Parasitol. Res., 107: 1323 - 1327. DOI: 10.1007/s00436-010-2004-x

Guardone, L., Schnyder, M., Macchioni, F., Deplazes, P., Magi, M. (2013): Serological detection of circulating Angiostrongylus vasorum antigen and specific antibodies in dogs from central and northern Italy. Vet. Parasitol., 192: 192 - 198. DOI: 10.1016/j.vetpar.2012.10.016

Guilhon, J., Cens, B. (1969): [Migrations and evolution of Angiostrongylus vasorum (Baillet, 1866) in dogs]. Comptes Rendus Hebdomadaires des Seances de l'Academie des Sciences - D: Sciences Naturelles, 269: 2377 - 2380

Hajnalová, M., JuRÁnKová, J., Svobodová, V. (2014): Dog's lungworm disease in the Czech Republic. In: E. Fók AND I. Kucsera (Eds) Fourth European Dirofilaria and Angiostrongylus Days (FEDAD), Budapest, Hungary, July 3 - 4, 2014. Program and abstracts, p. 103

HurníkovÁ, Z., Miterpáková, M., Mandelík, R. (2013): First autochthonous case of canine Angiostrongylus vasorum in Slovakia. Parasitol. Res., 112 (10): 3505 - 3508. DOI: 10.1007/s00436-013-3532-y KoCH, J., Willesen, J.L. (2009): Canine pulmonary angiostrongylosis: An update. Vet. J., 179 (3): 348 - 359. DOI: 10.1016/j. tvjl.2007.11.014

KoCH, J., Willesen, J.L., Jessen, I.R. (2005): Angiostrongylus vasorum (French heartworm) in dogs - epidemiological and clinical aspects. In: Proceedings of the International Conference on Emerging Snail Borne Helminth Diseases. Copenhagen; Denmark, 2005, pp. $10-12$

Maier, K., Löwenstein, M., Duscher, G., Leschnik, M., Joachim, A. (2010): Angiostrongylus vasorum, der Französische Herzwurm: auch ein Problem in Österreich? Wien. Tierarztl. Monatsschr., 97 (7-8): $171-180$

Majoros, G., FukÁr, O., Farkas, R. (2010): Autochthonous infection of dogs and slugs with Angiostrongylus vasorum in Hungary. Vet. Parasitol., 174 (3 - 4): 351 - 354. DOI: 10.1016/j.vetpar.2010.09.012 
Miterpáková, M., Iglódyová, A., Hurníková, Z. (2012): Canine subcutaneous dirofilariosis - noteless and neglected. Difficulties in disease monitoring. Helminthologia, 49 (4): 225 - 228. DOI 10.2478/s 11687-012-0042-x

Miterpáková, M., Hurníková, Z., Zalewski, A.P. (2014): The first clinically manifested case of angiostrongylosis in a dog in Slovakia. Acta Parasitol., 59 (4): 661 - 665. DOI: 10.2478/s11686-0140289-0

Morgan, E.R., Jefferies, R., Krajewski, M., Ward, P., Shaw, S.E. (2009): Canine pulmonary angiostrongylosis: the influence of climate on parasite distribution. Parasitol. Int., 58: 406 - 410. DOI: 10.1016/j.parint.2009.08.003

Mozzer, L.R., LIMA, W.S. (2015): Gallus gallus domesticus: Paratenic host of Angiostrongylus vasorum. Vet. Parasitol., 207: 81 - 84. DOI: 10.1016/j.vetpar.2014.11.003

Schnyder, M., Fahrion, A., Riond, B., Ossent, P., Webster, P., Kranjc, A., Glaus, T., Deplazes, P. (2010): Clinical, laboratory and pathological findings in dogs experimentally infected with $A n-$ giostrongylus vasorum. Parasitol. Res., 107: 1471 - 1480. DOI: 10.1007/s00436-010-2021-9

Schnyder, M., Tanner, I., Webster, P., Barutzki, D., Deplazes, P. (2011): An ELISA for sensitive and specific detection of circulating antigen of Angiostrongylus vasorum in serum samples of naturally and experimentally infected dogs. Vet. Parasitol., 179 (1 - 3): 152 - 158. DOI: 10.1007/s00436-013-3285-7

Schnyder, M., Schaper, R., Bilbrough, G., Morgan, E.R., Deplazes, $P$. (2013a): Seroepidemiological survey for canine angiostrongylosis in dogs from Germany and the UK using combined detection of Angiostrongylus vasorum antigen and specific antibodies. Parasitology, 140: 1442 - 1450. DOI: 10.1017/S0031182013001091 Schnyder, M., Schaper, R., Pantchev, N., Kowalska, D., Szwedko, A., Deplazes, P. (2013b): Serological detection of circulating Angiostrongylus vasorum antigen- and parasite-specific antibodies in dogs from Poland. Parasitol. Res., 112 (Suppl 1): 109-117. DOI: 10.1007/s00436-013-3285-7
Schnyder, M., Stebler, K., Naucke, T.J., Lorentz, S., Deplazes, P. (2014): Evaluation of a rapid device for serological in-clinic diagnosis of canine angiostrongylosis. Parasit. Vectors, 7: 72. DOI:10.1186/1756-3305-7-72

Schucan, A., Schnyder, M., Tanner, I., Barutzki, D., Traversa, D., Deplazes, P. (2012): Detection of specific antibodies in dogs infected with Angiostrongylus vasorum. Vet. Parasitol., 185 (2 - 4): 216 - 224. DOI: 10.1016/j.vetpar.2011.09.040

Simin, S., Spasojević Kosić, L., Kuruca, L., Pavlović, I., Savović, M., LALOŠEVIĆ, V. (2014): Moving the boundaries to the South-East: first record of autochthonous Angiostrongylus vasorum infection in a dog in Vojvodina province, northern Serbia. Parasit. Vectors, 7: 396. http://www.parasitesandvectors.com/content/7/1/396. DOI:10.1186/1756-3305-7-396

Sréter, T., Szell, Z., Marucci, G., Pozio, E., Varga, I. (2003): Extraintestinal nematode infections of red foxes (Vulpes vulpes) in Hungary. Vet. Parasitol., 115: 329 - 334

Staebler, S., Ochs, H., Steffen, F., Naegeli, F., Borel, N., SieBer-Ruckstuhl, N., Deplazes, P. (2005): Autochthonous infections with Angiostrongylus vasorum in dogs in Switzerland and Germany (in German). Schweizer Archiv fur Tierheilkunde, 147: 121 127

Szczęsna, J., Popiolek, M., Śmietana, W. (2007): A study on the helminthofauna of wolves (Canis lupus L.) in the Bieszczady Mountains (south Poland - preliminary results) (in Polish). Wiad. Parazytol., 53 (Suppl.): 36

Takacs, A., Szabo, L., Juhasz, L., Takacs, A.A., LanszKI, J., TAKacs, P.T., HELTAI, M. (2014): Data on the parasitological status of golden jackal (Canis aureus L., 1758) in Hungary. Acta Vet. Hung., 62: 33 - 41. DOI: 10.1556/AVet.2013.058

Tolnal, Z., Szell, Z., SRETer, T. (2015): Environmental determinants of the spatial distribution of Angiostrongylus vasorum, Crenosoma vulpis and Eucoleus aerophilus in Hungary. Vet. Parasitol., 207: 355 - 358. DOI: 10.1016/j.vetpar.2014.12.008 